\title{
Positronium Formation at Low Temperatures in Polymers and Other Molecular Solids
}

\author{
T. HIRADE \\ Department of Materials Science, Japan Atomic Energy Research Institute \\ 2-4, Shirakata Shirane, Tokai, Naka, Ibaraki, 319-1195, Japan \\ Positronium (Ps) formation by weakly localized long-lived electrons \\ (trapped electrons or anions) and positrons at low temperatures success- \\ fully explained the Ps formation enhancement at low temperatures observed \\ in polymers and molecular solids. This Ps formation process is different \\ from Ore process and spur process. The phenomena expected by this Ps \\ formation mechanism, such as an effect due to visible light exposure, a den- \\ sity dependence of weakly localized electrons and the delayed Ps formation \\ were successfully observed as the experimental evidence of this Ps formation \\ process. Some possibilities for application of this new Ps formation were \\ also given. One is a new idea for estimation of spin polarity of positrons. \\ Another is the possibility of application to study the local molecular motion \\ by observing the decay of Ps formation intensity.
}

PACS numbers: 36.10.Dr, 78.70.Bj, 36.20.Kr

\section{Introduction}

It has been reported that the yield of positronium (Ps) formation at low temperatures increases gradually during positron annihilation lifetime (PAL) measurements in materials such as polymers and molecular solids since 1980's [1, 2]. It was believed that the slow increment of Ps formation was caused by a change of physical properties of polymers, such as the physical relaxation of polymer chains until 1998. However, it has been clarified that the slow increment is caused by the reaction of free positrons and long-lived weakly localized electrons accumulated slowly with positron irradiation during PAL measurement by observing several expected phenomena [3-5]. Figure 1 is the first experimental evidence of the Ps formation by the reaction of positrons and long-lived weakly localized electrons. 
Here, more experimental results and details of the Ps formation process by the reaction of positrons and long-lived weakly localized electrons will be introduced.

\section{Trapped electrons and anions}

Ionizing radiation gives energy to materials by forming excited states or ion-excess electron pairs. If temperature of the sample is low enough and there are suitable sites for electron localization, some of the excess electrons are localized and live for long.

There are some states of long-lived weakly localized electrons, like trapped electrons (mainly localized between molecules) and anions (localized on molecules). Electrons are mainly localized as trapped electrons in polyethylene, and as anions in poly(methyl methacrylate) (PMMA). To identify the mechanism of electron localization, for example, electron paramagnetic resonance (EPR) can be used [5]. In the case of trapped electrons, there will be small interactions between trapped electrons and surrounding molecules. Therefore the $g$-factor of the trapped electrons should be quite close to the $g$-factor of free electrons, 2.0023. Relaxation time is also long and power saturation easily occurs on EPR measurement. For anions, the interaction between electrons and molecules are large and then the $g$-factor will be shifted and EPR spectra often have structures [5]. The depth of potential for electrons is several $\mathrm{eV}$, which corresponds to the energy of visible light. Therefore these shallowly localized electrons give visible color on samples.

These electrons are released by elevating the temperature of the sample, because the molecular motions cause dynamic modifications of the trapping potentials. This means that the decay of the weakly localized electrons gives information of the molecular motions, such as the apparent activation energy [6]. There are several methods to see the weakly localized electrons, such as EPR, light absorption, glow curve measurements, and now positron techniques can also be good tools to observe the weakly localized electrons. Moreover, the positron techniques seem to have some advantages [6].

It is well known in the field of radiation chemistry that impurity can provide suitable localizing sites for electrons. Hence, it is quite natural that polymers produced at different place can give different results.

The frozen molecular motions can provide the suitable localizing sites. Therefore there can be several localization sites in one polymer. For example, in the case of PMMA, a complicated phenomenon was observed [7]. It is a good example that there are, at least, two localizing sites for electrons. One is related to the rotational motion of methyl group and the other is, probably, local motion of main chain [6]. Existing of the two localizing sites for electrons in PMMA were also reported by the use of the glow curve measurement [8]. He et al. indicated that there are two electron trapping sites in polyethylene [9]. 


\section{Ps enhancement at low temperatures}

Positrons have a chance to form Ps in the positron spur, the terminal spur of the positron track. Experimental results have been well explained by the spur reaction model proposed by Mogensen (see p. 66 and p. 112 in Refs. [10, 11]). Positrons and excess electrons play important roles in this model. This model can explain almost all of the results with the knowledge of radiation chemistry. Sometimes the spur model can give new information for radiation chemistry (see p. 129 of Refs. [10, 12]). According to the spur reaction model, it was believed that positrons escaped from Ps formation in the positron spur must annihilate as free positrons with a lifetime of about $400 \mathrm{ps}$.

In 1998, a new Ps formation process was proposed [3, 4]. High-energy positrons injected in samples create excess electrons and some of them will localize and live for long time at low enough temperatures, because local molecular motions are frozen and can provide suitable localizing sites for electrons. The binding energy of these localized electrons is usually $0.5-3 \mathrm{eV}$. The binding energy of $\mathrm{Ps}$ is $6.8 \mathrm{eV}$ in vacuum and probably about $4-5 \mathrm{eV}$ in condensed materials such as polymers and molecular solids. Hence, free positrons can pick up these localized electrons to form Ps.

The typical binding energy of weakly localized electrons is almost the same as visible light energy, therefore visible light can quench the localized electrons in many cases. Hence, visible light should affect the increment of Ps formation. The first experimental result of this is shown in Fig. 1 [3, 5]. Later, the KEK group did a similar experiment for polyethylene and also found the visible light effect [13].
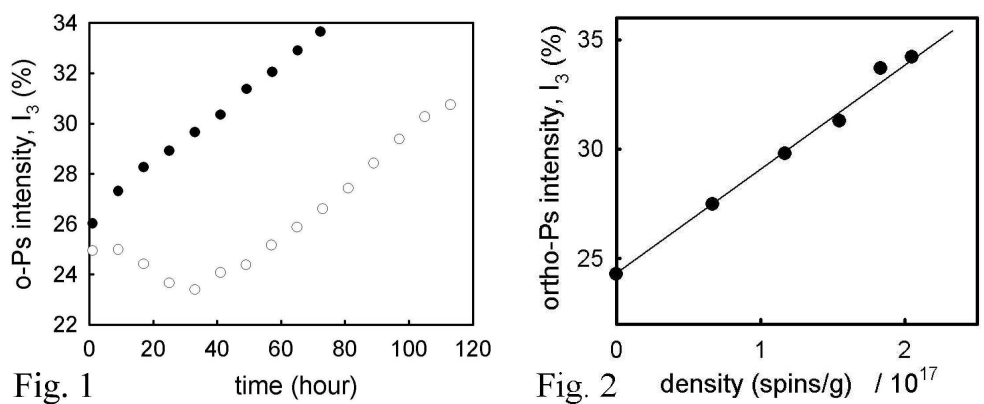

Fig. 1. Positron annihilation lifetime measurement. The time dependence of the intensity of $o$-Ps, $I_{3}$, in PMMA6N at $120 \mathrm{~K}$ [4]. (• in darkness, o with visible light).

Fig. 2. The relation between the density of anions (shallowly localized electrons) and the intensity of ortho-Ps, $I_{3}$, at $77 \mathrm{~K}$ in PMMA6N [4].

During PAL measurements, positrons irradiate the sample continuously. Therefore the effect of irradiation will be accumulated. When the measurement temperature is low enough that the weakly localized electrons can live for long time, 
the density of the weakly localized electrons will increase with elapsed measurement time. With the higher density of weakly localized electrons, the probability of Ps formation by the reaction of free positrons and the localized electrons is larger. There is a linear relation between the density of weakly localized electrons and yield of Ps formation, as shown in Fig. 2 [5]. Here $\gamma$-irradiation from outside of the samples was applied instead of positron irradiation.

\section{Delayed Ps formation by free positrons and weakly localized electrons}

Brusa et al. indicated that the diffusion length of positrons was very large in polyethylene [14]. It was $c a .0 .2 \mathrm{~cm}^{2} / \mathrm{s}$ at around $77 \mathrm{~K}$. It was measured by the shift of Doppler peak with applying an external electric field. If positrons are localized somewhere before annihilation, it is impossible to measure the mobility by this method. It means that some of free positrons can move freely even until they annihilate. There is some experimental evidence of Ps formation by the reaction of free positrons and localized electrons at low temperatures. These two facts suggest that Ps formation is possible even at a positron age of $300 \mathrm{ps}$ in polyethylene. The age momentum correlation (AMOC) technique has been applied to investigate delayed Ps formation.

Positron-electron pair annihilation $\gamma$-rays are close to $511 \mathrm{keV}$, in the case of annihilation with two-photon emission. The shift from $511 \mathrm{keV}$, the Doppler shift, is caused by the sum of the momenta of the positron and the electron. For just $p$-Ps, the sum of the momenta is almost zero. Hence the change of the shape of $511 \mathrm{keV}$ peak, Doppler broadening, can give information about $p$-Ps formation. The peak shape parameter of $511 \mathrm{keV}$ annihilation $\gamma$-rays, the $S$-parameter, is often used. The formation of more $p$-Ps, i.e. a sharper peak, gives a larger $S$-parameter.

At the early age of a positron, up to $0.6 \sim 0.7 \mathrm{~ns}$, a large $S$-parameter appears because of the annihilation of $p$-Ps. For no weakly localized electrons, the largest $S$-parameter appears just after the time zero. On the other hand, while there are a lot of weakly localized electrons, an increase in the $S$-parameter appears just after time zero and the maximum value appears around $0.3 \mathrm{~ns}$. Delayed Ps formation was clearly observed by AMOC measurements [15].

\section{Localized electrons as a probe}

Before 1998, people believed that the large increment and decrement of Ps formation in polymers at low temperatures could give some information about physical properties of polymers $[16,17]$. However this phenomenon was caused by the weakly localized electrons. 
Decay of trapped electrons or anions by elevating the temperature can give information about molecular motions, because the molecular motions cause dynamic modifications of the trapping potentials. The variation of $o$-Ps formation, $I_{3}$, at low temperatures can give information about local molecular motions. It is possible to obtain the apparent activation energy, $E_{m}$, by PAL measurements at several temperatures, $T$, via Eq. (1) [6]

$$
\frac{E_{m}}{R T}=\log \left(\frac{A}{k_{i r}}\right)+\log \left(\frac{I_{3}(T)-I_{3 L}}{I_{3 m}-I_{3}(T)}\right) .
$$

Here, $I_{3}(T)$ is the stable intensity of $o$-Ps in darkness, $I_{3 m}$ is the maximum stable intensity and $I_{3 L}$ is $o$-Ps intensity with no localized electrons. $k_{i r}$ and $A$ are, probably, almost constant at the temperature range where a large decrement of $I_{3}$ occurs. $R$ is Boltzmann's constant.

A positron is a strong tool to investigate weakly localized electrons, because it is possible to probe these electrons, even if EPR or light absorption measurements cannot detect them [6].

\section{A new positron polarimeter}

There is one big difference between Ps formations by the spur process and by the reaction of positrons and weakly localized electrons. For Ps formation by the spur process, spins of the electrons that will react with positrons to form Ps are randomly distributed. However, for Ps formation by the reaction of positrons and weakly localized electrons, spins of the electrons can be polarized in magnetic field. This is the reason why the localized electrons are observable by EPR measurement. If the temperature is very low and the magnetic field is very high, the spin polarity of the weakly localized electrons is large enough to see the effect on Ps formation by spin polarized positrons and spin polarized electrons.

Ps has two ground states, $p$-Ps and $o$-Ps, because of the difference of the spin states. $p$-Ps and the $m=0$ substate of $o$-Ps are perturbed by the existence of a static magnetic field. Perturbed Ps states are noted as $p^{\prime}$-Ps and $o^{\prime}$-Ps here.

Here, $P$ is the polarity of positrons and $E$ is the polarity of electrons along the magnetic field $B$. The definition of the polarity of positrons $P$ (and electrons $E$ ) is

$$
P(E)=\frac{N(\uparrow)-N(\downarrow)}{N(\uparrow)+N(\downarrow)}
$$

where $N(\uparrow)$ is the population of the positrons (electrons) in the upper spin state, and $N(\downarrow)$ is the population of the positrons (electrons) in the lower spin state.

The expected spin states of Ps are shown in Table I. The formation probabilities of $o^{\prime}$-Ps and $p^{\prime}$-Ps, $F_{o^{\prime}}$ and $F_{p^{\prime}}$, respectively, in a magnetic field $B$ are:

$$
F_{o^{\prime}}=\frac{(1+y)^{2}(1-P)(1+E)+(1-y)^{2}(1+P)(1-E)}{8\left(1+y^{2}\right)}
$$




$$
F_{p^{\prime}}=\frac{(1-y)^{2}(1-P)(1+E)+(1+y)^{2}(1+P)(1-E)}{8\left(1+y^{2}\right)},
$$

where $y$ is given by $y=x /\left[\left(1+x^{2}\right)^{1 / 2}+1\right]$ with $x=4 \mu B / \kappa \hbar \omega_{0}$. $P$ is the positron spin polarity and $E$ is the spin polarity of weakly localized electrons along $B . \mu$ is the magnetic moment of the electron and $\hbar \omega_{0}$ is the hyperfine structure splitting between $o$-Ps and $p$-Ps in vacuum; $\kappa$ is the electron-positron contact density.

TABLE I

Fractions of Ps spin states at the opposite magnetic field directions. $\uparrow_{p}\left(\downarrow_{p}\right)$ indicates the spin state of positron and $\uparrow_{\mathrm{e}}\left(\downarrow_{\mathrm{e}}\right)$ indicates the spin state of electron.

\begin{tabular}{c|cc|cc|ccc}
\hline \hline Angle $\left[^{\circ}\right]$ & $\begin{array}{c}\text { Positron } \\
\text { spin }\end{array}$ & Fraction & $\begin{array}{c}\text { Electron } \\
\text { spin }\end{array}$ & Fraction & $\begin{array}{c}\text { Ps spin } \\
\text { state }\end{array}$ & Fraction \\
\hline \multirow{3}{*}{0} & $\uparrow_{\mathrm{p}}$ & $(1+P) / 2$ & $\uparrow_{\mathrm{e}}$ & $(1+E) / 2$ & $\left|\uparrow_{\mathrm{p}}, \uparrow_{\mathrm{e}}\right\rangle$ & $(1+P)(1+E) / 4$ \\
\cline { 4 - 7 } & & $\downarrow_{\mathrm{e}}$ & $(1-E) / 2$ & $\mid \uparrow_{\mathrm{p}}$, & $\left.\downarrow_{\mathrm{e}}\right\rangle$ & $(1+P)(1-E) / 4$ \\
\cline { 4 - 7 } & $\downarrow_{\mathrm{p}}$ & $(1-P) / 2$ & $\uparrow_{\mathrm{e}}$ & $(1+E) / 2$ & $\left|\downarrow_{\mathrm{p}}, \uparrow_{\mathrm{e}}\right\rangle$ & $(1-P)(1+E) / 4$ \\
\cline { 4 - 7 } & & $\downarrow_{\mathrm{e}}$ & $(1-E) / 2$ & $\mid \downarrow_{\mathrm{p}}$, & $\left.\downarrow_{\mathrm{e}}\right\rangle$ & $(1-P)(1-E) / 4$ \\
\hline \multirow{3}{*}{180} & \multirow{2}{*}{$\uparrow_{\mathrm{p}}$} & $(1-P) / 2$ & $\uparrow_{\mathrm{e}}$ & $(1+E) / 2$ & $\mid \uparrow_{\mathrm{p}}$, & $\left.\uparrow_{\mathrm{e}}\right\rangle$ & $(1-P)(1+E) / 4$ \\
\cline { 4 - 7 } & & & $\downarrow_{\mathrm{e}}$ & $(1-E) / 2$ & $\mid \uparrow_{\mathrm{p}}$, & $\left.\downarrow_{\mathrm{e}}\right\rangle$ & $(1-P)(1-E) / 4$ \\
\cline { 4 - 7 } & \multirow{2}{*}{$\downarrow_{\mathrm{p}}$} & $(1+P) / 2$ & $\uparrow_{\mathrm{e}}$ & $(1+E) / 2$ & $\mid \downarrow_{\mathrm{p}}$, & $\left.\uparrow_{\mathrm{e}}\right\rangle$ & $(1+P)(1+E) / 4$ \\
\cline { 3 - 7 } & & & $\downarrow_{\mathrm{e}}$ & $(1-E) / 2$ & $\mid \downarrow_{\mathrm{p}}$, & $\left.\downarrow_{\mathrm{e}}\right\rangle$ & $(1+P)(1-E) / 4$
\end{tabular}

The self-annihilation rates of $o^{\prime}$-Ps and $p^{\prime}$-Ps in a magnetic field, $\Gamma_{o^{\prime}}$ and $\Gamma_{p^{\prime}}$, respectively, are:

$$
\begin{gathered}
\Gamma_{p^{\prime}}=\kappa \frac{y^{2} \gamma_{o}+\gamma_{p}}{1+y^{2}}, \\
\Gamma_{o^{\prime}}=\kappa \frac{\gamma_{o}+y^{2} \gamma_{p}}{1+y^{2}},
\end{gathered}
$$

where $\gamma_{o}$ and $\gamma_{p}$ are the self-annihilation rates of $o$-Ps and $p$-Ps in vacuum. The self-annihilation rate of the $o^{\prime}$-Ps component in a magnetic field of $400 \mathrm{mT}$ is about $0.00304 \mathrm{~ns}^{-1}$ with $\kappa=1$. It is much smaller than the Ps pickoff annihilation rate that is about $1 \mathrm{~ns}^{-1}$. At $2 \mathrm{~T}$, it will be $0.467 \mathrm{~ns}^{-1}$.

Polarization of weakly localized electrons in a $400 \mathrm{mT}$ magnetic field is about -0.00349 at $77 \mathrm{~K},-0.0639$ at $4.2 \mathrm{~K}$, and -0.204 at $1.3 \mathrm{~K}$. At higher magnetic fields and lower temperatures, the effect will be larger. For example, $E$ will be -0.309 at $4.2 \mathrm{~K}$ and -0.775 at $1.3 \mathrm{~K}$ in $2 \mathrm{~T}$ magnetic field. We are assuming the polarity of positrons as 0.335 , the polarity of positrons emitted for a half of total solid angle, $2 \pi$, from ${ }^{22} \mathrm{Na}$. 
The expected yields of perturbed states calculated by Eqs. (3) and (4), and of unperturbed states obtained with the equations in Table I are shown in Table II. The total yields of long-lived unperturbed $o$-Ps are $46.6(26.6+20.0) \%$ and 53.4 $(13.2+40.2) \%$ for angles of $0^{\circ}$ and $180^{\circ}$. The difference is $6.8 \%$. As mentioned above, at lower temperatures and higher magnetic fields, the effect will be larger.

\section{TABLE II}

The formation probabilities of every state by turning the magnetic field of $400 \mathrm{mT}$ at $1.3 \mathrm{~K}$.

\begin{tabular}{c|c|c}
\hline \hline Angle $\left[^{\circ}\right]$ & \multicolumn{2}{|c}{ Ps } \\
\hline \multirow{4}{*}{0} & $o$-Ps $(m=1)$ & 0.266 \\
& $p^{\prime}$-Ps & 0.282 \\
& $o^{\prime}$-Ps & 0.253 \\
& $o-P s(m=-1)$ & 0.200 \\
\hline \multirow{4}{*}{180} & $o-P s(m=1)$ & 0.132 \\
& $p^{\prime}-\mathrm{Ps}$ & 0.229 \\
& $o-\operatorname{Ps}(m=-1)$ & 0.236 \\
& $o .402$
\end{tabular}

For high-density polyethylene (HDPE), Ps formation yield at $77 \mathrm{~K}$ is about $12 \%$ without weakly localized electrons and about $32 \%$ with them [5]. It means that Ps formation by weakly localized electrons is $20 \%$ in HDPE. It is $42 \%$ for cyclohexane [5]. Applying these values, we can estimate the expected change in the longest lifetime component, i.e. unperturbed $o$-Ps components, at $1.3 \mathrm{~K}$ in a $400 \mathrm{mT}(2 \mathrm{~T})$ magnetic field by changing the magnetic field. For HDPE this is $1.36(5.18) \%$ and for cyclohexane $2.86(10.9) \%$ for a positron polarity, $P$, of 0.335 .

In the $400 \mathrm{mT}$ magnetic field, it is difficult to separate $o$-Ps and $o^{\prime}$-Ps, as the self-annihilation rate of the $o^{\prime}$-Ps component is much smaller than the pickoff annihilation rate of Ps. However, the effect was observed by observing the change of the counts at the longer age region on the PAL spectra [18]. It will be quite possible with a very high magnetic field, such as $2 \mathrm{~T}$, in which the $o^{\prime}$-Ps self-annihilation rate is very large and the electron spin polarity is also very large. This method will be able to be a powerful tool to observe positron spin polarity.

\section{Conclusion}

In 1998, a new idea of Ps formation at low temperatures was proposed. Free positrons escaping from Ps formation in the positron spur can find trapped electrons or anions to form Ps at low temperatures. Some interesting phenomena predicted by the idea were successfully observed by experiments, such as a visible light effect, a density dependence of weakly localized electrons, and delayed Ps 
formation [5, 15]. All of these experimental results were good evidence of the new Ps formation, and these can be a good starting point for future research. There are many possibilities, such as observing local motions of molecular solids by measuring $I_{3}$ variation [6]. It has been shown that there is also a possibility to apply this new Ps formation reaction to see positron spin polarity.

\section{Acknowledgment}

The author acknowledges gratefully the valuable advice of Dr Morten Eldrup and Prof. Frans H.J. Maurer. He also wishes to express his appreciation for the supports by Mr Niels J. Pedersen, Dr Takayuki Kumada, and Dr Takenori Suzuki.

\section{References}

[1] M. Eldrup, D. Lightbody, J. Sherwood, Faraday Discussions 69, 175 (1980).

[2] P. Kindl, G. Reiter, Phys. Status Solidi A 104, 707 (1987).

[3] T. Hirade, C.L. Wang, F.H.J. Maurer, M. Eldrup, N.J. Pedersen, in: the 35th Annual Meeting on Radioisotopes in the Physical Science and Industries, JuneJuly 1998, Tokyo (Japan), Abstracts, p. 89.

[4] C.L. Wang, T. Hirade, F.H.J. Maurer, M. Eldrup, N.J. Pedersen, J. Chem. Phys. 108, 4654 (1998).

[5] T. Hirade, F.H.J. Maurer, M. Eldrup, Radiat. Phys. Chem. 58, 465 (2000).

[6] T. Hirade, Radiat. Phys. Chem. 68, 375 (2003).

[7] M. Eldrup, T. Hirade, N.J. Pedersen, F.H.J. Maurer, A. Lund, in: 7th Intern. Conf. on Positron and Positronium Chemistry, Knoxville (TN, U.S.A.) 2002, Abstracts, p. 105.

[8] R.J. Fleming, J. Polymer Science, Part A-2 6, 1283 (1968).

[9] C. He, T. Suzuki, L. Ma, M. Matsuo, V.P. Shantarovich, K. Kondo, Y. Ito, Phys. Lett. A 304, 49 (2002).

[10] O.E. Mogensen, Positron Annihilation in Chemistry, Springer, Berlin 1995.

[11] O.E. Mogensen, J. Chem. Phys. 60, 9983 (1974).

[12] N. Gee, G.R. Freeman, J. Chem. Phys. 90, 5399 (1989).

[13] Y. Ito, T. Hirade, E. Hamada, T. Suzuki, Y. Ito, Acta Phys. Pol. A 95, 533 (1999).

[14] R.S. Brusa, M. Duarte Naia, D. Margoni, A. Zecca, Appl. Phys. A 60, 447 (1995).

[15] N. Suzuki, T. Hirade, F. Saito, T. Hyodo, Radiat. Phys. Chem. 68, 647 (2003).

[16] T. Suzuki, Y. Oki, M. Numajiri, T. Miura, K. Kondo, N. Oshima, Y. Ito, Polymer 37, 5521 (1996).

[17] A. Uedono, T. Kawano, S. Tanigawa, M. Ban, M. Kyoto, T. Uozumi, J. Polym. Sci. B, Polym. Phys. 35, 1601 (1997).

[18] T. Hirade, T. Kumada, Mater. Sci. Forum 445-446, 301 (2004). 